\title{
EFEITO DO NÚMERO DE SEMENTES E DO VOLUME DE ÁGUA NA CONDUTIVIDADE ELÉTRICA DE SEMENTES DE Dalbergia nigra (Vell.) Fr.All. ex Benth. ${ }^{1}$
}

\author{
MARCO ANTONIO MARQUES ${ }^{2}$, RINALDO CESAR PAULA ${ }^{3}$ E TERESINHA JESUS DELÉO RODRIGUES ${ }^{4}$
}

\begin{abstract}
RESUMO - O presente trabalho teve como objetivos verificar o efeito do número de sementes e do volume de água utilizada no teste de condutividade elétrica (CE) para avaliar o vigor de três lotes de sementes de Dalbergia nigra (jacarandá-da-bahia) e correlacionar esses resultados com os dados de germinação em laboratório e em viveiro. Os testes de germinação em laboratório e viveiro foram conduzidos com quatro repetições de 25 sementes. O teste de $\mathrm{CE}$ foi realizado com 25,50 e 75 sementes embebidas a 75, 100 e $125 \mathrm{ml}$ de água, por diferentes períodos. A porcentagem de germinação e de plântulas normais em laboratório, indicaram o lote III como de qualidade inferior aos lotes I e II. A primeira contagem da germinação e o índice de velocidade de germinação em laboratório e a emergência, índice de velocidade de emergência e porcentagem de plântulas normais em viveiro identificaram o lote II como superior ao lote I e o III como inferior. A CE diminui com o aumento do volume de água e aumentou com o período de embebição. A diferenciação dos lotes foi mais eficiente, quando se utilizou $75 \mathrm{ml}$ de água deionizada e amostras de 50 sementes com pelo menos 36 horas de embebição, com valores de CE menores nos lotes I e II do que no lote II. Os coeficientes de correlação simples entre a CE e as demais características avaliadas, em laboratório e viveiro, foram elevados e significativos, evidenciando alta associação entre os mesmos. Assim, pode-se recomendar que o teste de $\mathrm{CE}$ seja conduzido a $25^{\circ} \mathrm{C}$, com amostras de 50 sementes embebidas em $75 \mathrm{ml}$ de água deionizada, por períodos iguais ou superiores a 36 horas de embebição, para determinar a qualidade fisiológica de lotes de sementes de jacarandá-da-bahia.

Termos para indexação: Dalbergia nigra, jacarandá-da-bahia, condutividade elétrica, qualidade fisiológica.
\end{abstract}

\section{SEED NUMBER AND WATER VOLUME EFFECTS ON THE ELECTRICAL CONDUCTIVITY TEST OF Dalbergia nigra (Vell.) Fr.All. ex Benth. SEEDS}

\begin{abstract}
This work was carried out with objective to verify the effect of Dalbergia nigra seeds number and water volume in the electrical conductivity test (EC) and the correlation this results with germination datas in laboratory and nursery. The germination tests were conduced with four replications of 25 seeds. The EC was conduced with 25,50 and 75 seeds imbibited in 75, 100 and $125 \mathrm{ml}$ of water by diferent period. Lot III showed lower physiological quality than lots I and II, considering the results obtained in the laboratory and in the nursery. The EC decreased with the increase of the volume of water and increased with the imbibition period. Lots I and II presented EC values lower than lot III, when the water volume was $75 \mathrm{ml}$ and 50 seeds were used; the differentiation of lots was more efficient when the imbibition period was, at least, of 36 hours. The simple correlation coefficients between $\mathrm{EC}$ and the characteristics evaluated under lab and nursery conditions, evidenced that the EC test was efficient to distinguish the physiological quality of the studied seed lots because presented high association between them. The EC test can
\end{abstract} ${ }^{1}$ Aceito para publicação em 31.12.2001; trabalho realizado com apoio do
CNPq.
${ }^{2}$ Eng ${ }^{\circ}$ Agro $^{\circ}$, MSc. em Produção e Tecnologia de Sementes; e-mail:
prosbo@ig.com.br

\footnotetext{
3 Eng $^{\circ}$ Florestal, Doutor em Ciências Florestais, Prof. do Depto. Produção Vegetal, FCAV/UNESP, 14884-900, Jaboticabal-SP; e-mail: rcpaula@fcav. unesp.br

${ }^{4}$ Biológa, PhD., Prof do Depto. Biologia Aplicada a Agropecuária, FCAV/ UNESP; e-mail: tdelro@fcav.unesp.br
} 
be used to establish the physiological quality of Dalbergia nigra seed lots. It can be conducted at $25^{\circ} \mathrm{C}$, with samples of 50 seeds and $75 \mathrm{ml}$ of deionized water, and imbibition periods equal or longer than 36 hours.

Index terms: Dalbergia nigra, jacarandá-da-Bahia, electrical conductivity, physiological quality.

\section{INTRODUÇÃO}

É de interesse prático, quando se dispõe de diferentes lotes de sementes, conhecer a qualidade fisiológica intrínseca a cada um. Sabe-se, contudo, que a queda na germinação é um dos últimos eventos que caracterizam o declínio na qualidade fisiológica de sementes e o teste de germinação, isoladamente, não é adequado para discriminar coretamente os lotes de sementes, pois, quando possuem porcentagem de germinação semelhantes, podem apresentar qualidades fisiológicas distintas (Bonner et al., 1994).

Tratando-se de espécies florestais, grande parte dos testes utilizados para determinar a qualidade fisiológica dos lotes, foram desenvolvidos a partir da utilização de sementes de espécies agrícolas, com alto grau de domesticação e melhoramento. Muitas vezes faz-se uso de testes de vigor, sem o ajuste adequado da metodologia de avaliação, de forma que os resultados obtidos podem não corresponder a verdadeira qualidade fisiológica dos lotes de sementes avaliados. A grande variabilidade existente, em termos de maturação e tamanho de sementes, dentre outros, entre as várias sementes que constituem um lote e no caso específico das espécies florestais nativas, faz com que a variação nos resultados de uma repetição para outra, num mesmo lote de sementes, reproduza resultados que devem ser trabalhados com cautela (Bonner, 1998).

$\mathrm{O}$ teste de condutividade elétrica, por ser fácil, rápido e capaz de identificar a deterioração das sementes em seu estádio inicial, tem grande potencialidade para sementes florestais, conforme constatado por Barbedo \& Cícero (1998), para sementes de Inga uruguensis Hook. et Arn., e sugerido por Bonner (1998) para sementes florestais em geral. O teste de condutividade elétrica foi utilizado por Ferraz et al. (1991) para avaliação do vigor de sementes de Carapa procera DC., porém os resultados obtidos não foram satisfatórios. Paula et al. (1997) reportaram que o teste de condutividade elétrica foi eficiente para verificar alterações fisiológicas em sementes de Hevea brasiliensis Muell.Arg. durante o armazena-mento. Bilia et al. (1998) utilizaram este teste com sucesso para avaliar o desempenho de sementes de Inga uruguensis em diferentes condições. Contudo, embora alguns resultados demonstrem a potencialidade deste teste, torna-se necessário o ajuste de sua metodologia para as várias espécies florestais. Este ajuste, além de propiciar resultados mais confiáveis, poderá reduzir consideravelmente o tempo de avaliação da qualidade fisiológica de lotes de sementes destas espécies que tem como fator complicador, para algumas delas, o longo tempo para completar a germinação.

Neste sentido, o presente trabalho teve como objetivos verificar a aplicabilidade e ajustar a metodologia do teste de condutividade elétrica para avaliar o vigor de sementes de $\mathrm{Dal}$ bergia nigra (jacarandá-da-bahia), no que se refere ao número de sementes e volume de água utilizada no teste, bem como correlacionar esses resultados com os dados de germinação em laboratório e em viveiro.

\section{MATERIAL E MÉTODOS}

Foram utilizadas sementes de Dalbergia nigra (Vell.) Fr. All. ex Benth. (jacarandá-da-bahia) pertencentes a três lotes (anos de colheita: lote I-1998, lote II-1997 e lote III-1994). Os lotes apresentavam idades e períodos de armazenamento diferentes e foram obtidas junto ao Laboratório de Análises de Sementes Florestais da Universidade Federal de Viçosa, Viçosa-MG.

Após colhidas, as sementes foram retiradas manualmente dos frutos, eliminando-se as sementes chochas e danifi-cadas. Depois foram armazenadas em tamboretes de fibra de madeira, em câmara fria $\left(5^{\circ} \mathrm{C}\right.$ e $85 \pm 5 \%$ de umidade relativa) até fevereiro de 2000, quando foram enviadas para a Faculdade de Ciências Agrárias e Veterinárias (FCAV/UNESP), Campus de Jaboticabal e encaminhadas ao Departamento de Biologia Aplicada à Agropecuária, onde foram armazenadas dentro de tamboretes de fibra de madeira, em câmara fria $\left(5-10^{\circ} \mathrm{C}\right.$ e $70-75 \%$ de UR), durante toda a fase experimental. Foram retiradas amostras de sementes de cada um dos lotes para determinar: teor inicial de água - pelo método da estufa a $105 \pm 3^{\circ} \mathrm{C}$ por 24 horas (Brasil, 1992), utilizando-se três repetições de 100 sementes; porcentagem e índice de velocidade de germinação - o teste foi realizado em caixas plásticas de germinação $(11 \mathrm{x} 11 \mathrm{~cm})$, desinfetadas com hipoclorito de sódio a $5 \%$ e revestidas com duas folhas de papel para germinação. 
Todo material, exceto as sementes e as caixas, foi autoclavado a $120^{\circ} \mathrm{C}$ por 20 minutos. $\mathrm{O}$ substrato foi umedecido com solução de nistatina a $0,2 \%$. Foram utilizadas quatro repetições de 25 sementes e o experimento foi conduzido em germinador vertical tipo $\mathrm{BOD}$, a $25^{\circ} \mathrm{C}$ constantes e fotoperíodo de oito horas. As contagens do número de sementes germinadas foram realizadas diariamente, sempre no mesmo horário, até o final do teste (11 dias), considerando-se como critério de germinação a protrusão da radícula $(2 \mathrm{~mm})$; primeira contagem da germinação - foi avaliada juntamente com o desenvolvimento do teste de germinação, sendo avaliada no sétimo dia após a instalação do teste; emergência e índice de velocidade de emergência em viveiro - para esse estudo foi utilizado como substrato um mistura à base de composto orgânico, casca de arroz carbonizada e terra de subsolo (na proporção de 6:3:1, v/v) e as sementes foram colocadas para germinar em casa de vegetação, com $50 \%$ de sombrea-mento e irrigação intermitente. A semeadura foi realizada em tubetes de plástico rígido com $50 \mathrm{~cm}^{3}$ de substrato, à profundidade aproximada de $1,0 \mathrm{~cm}$. A avaliação da emergência das plântulas foi realizada até sua estabilização, o que ocorreu com 23 dias. Avaliou-se a porcentagem final de emergência (E) e o índice de velocidade de emergência de plântulas (IVE), de acordo com Maguire (1962); durante o período experimental em viveiro, a temperatura média ambiental foi de $22,4^{\circ} \mathrm{C}$, a umidade relativa do ar de $73,5 \%$, a temperatura máxima de $29,4^{\circ} \mathrm{C}$ e a mínima de $17,1^{\circ} \mathrm{C}$; porcentagem de plântulas normais em laboratório e em viveiro - foi avaliada de acordo com Oliveira (1993), considerando-se como normais as plântulas com todas as estruturas essenciais perfeitas; teste de condutividade elétrica - avaliou-se o efeito do número de sementes por repetição $(25,50$ e 75 sementes $)$ e do volume de água de embebição $(75,100$ e $125 \mathrm{ml}$ de água deionizada) nos resultados do teste de condutividade elétrica, após 6,12 , $18,24,36$ e 72 horas de embebição das sementes, com quatro repetições por tratamento. Os resultados obtidos foram expressos em $\mu \mathrm{mol} / \mathrm{cm} / \mathrm{g}$.

Os resultados dos testes de germinação, em laboratório e em viveiro, foram submetidos a análise de variância, seguindo um delineamento inteiramente casualizado, com três tratamentos (três lotes) e quatro repetições de 25 sementes cada uma. Para o teste de condutividade elétrica, o delineamento experimental foi o inteiramente casualizado, em parcelas subdivididas. As parcelas foram distribuídas no esquema fatorial $3 \times 3 \times 3$ (três lotes de sementes; três quantidades $\left(\mathrm{n}^{\circ}\right)$ de sementes e três volumes de água deionizada) e as subparcelas foram os seis períodos de embebição, com quatro repetições por tratamento. As médias de lotes, número de sementes e volume de água foram comparadas pelo teste Tukey, a $5 \%$. Os graus de liberdade para a interação lote-número de sementes-volume de água-período de embebição foram decompostos pelo uso da análise de regressão polino-mial até terceiro grau. $\mathrm{O}$ modelo escolhido correspondeu aquele de maior grau que, na decomposição dos graus de liberdade, apresentou significância estatística a, pelo menos, $5 \%$ pelo teste F. Os resultados dos testes de condutividade elétrica foram correlacionados com os resultados dos testes de germinação em laboratório e viveiro, pela análise de correlação simples, a 5\% pelo teste F.

\section{RESULTADOS E DISCUSSÃO}

Na Tabela 1 encontram-se os resultados dos testes de germinação conduzidos em laboratório e viveiro. Verifica-se pelos resultados da primeira contagem da germinação (PC) e índice de velocidade de germinação (IVG) e pelas avalia-

\begin{tabular}{|c|c|c|c|c|c|c|c|}
\hline \multirow{2}{*}{ Lotes } & \multicolumn{4}{|c|}{ Laboratório } & \multicolumn{3}{|c|}{ Viveiro } \\
\hline & $\mathrm{PC}(\%)$ & $\mathrm{G}(\%)$ & PNL (\%) & IVG & E $(\%)$ & PNV (\%) & IVE \\
\hline Lote I & $71,0 \quad b$ & $79,0 \mathrm{a}$ & $45,0 \mathrm{a}$ & $3,3 \mathrm{~b}$ & $51,5 \mathrm{~b}$ & $34,0 \quad b$ & $0,70 \mathrm{~b}$ \\
\hline Lote II & $88,0 \mathrm{a}$ & $90,0 \mathrm{a}$ & $68,0 \mathrm{a}$ & $4,4 \mathrm{a}$ & $79,2 \mathrm{a}$ & $72,6 \mathrm{a}$ & $1,20 \mathrm{a}$ \\
\hline Lote III & $14,0 \quad \mathrm{c}$ & $21,0 \quad b$ & $13,0 \mathrm{~b}$ & $0,7 \quad \mathrm{c}$ & $7,5 \quad \mathrm{c}$ & $4,5 \quad \mathrm{c}$ & $0,08 \quad \mathrm{c}$ \\
\hline Valor de F & $94,55 * *$ & $95,15 * *$ & $6,04^{*}$ & $120,40 * *$ & $46,72 * *$ & $29,35^{*}$ & $52,74 * *$ \\
\hline CV $(\%)$ & 13,82 & 12,00 & 51,68 & 12,10 & 22,97 & 34,45 & 23,35 \\
\hline Média & 57,67 & 63,33 & 33,67 & 2,82 & 46,08 & 32,82 & 0,66 \\
\hline \multicolumn{8}{|c|}{$\begin{array}{l}\text { ** Valor significativo a } 1 \% \text { pelo teste } \mathrm{F} \text {. } \\
\text { Médias seguidas pela mesma letra, na coluna, não diferem entre si pelo teste Tukey, a } 1 \% . \\
\mathbf{P C}=\text { Primeira contagem da germinação; } \mathbf{G}=\text { Germinação; } \mathbf{P N L}=\text { Plântulas normais em laboratório; } \mathbf{I V G}=\text { Índice de velocidade de germinação; } \\
\text { = Emergência; } \mathbf{P N V ~ = ~ P l a ̂ n t u l a s ~ n o r m a i s ~ e m ~ v i v e i r o ; ~} \mathbf{I V E}=\text { Índice de velocidade de emergência. }\end{array}$} \\
\hline
\end{tabular}


ções em viveiro, que o lote II foi o de melhor qualidade, seguido pelo lote I e, por último pelo lote III que foi o mais velho, colhido em 1994. Contudo, ao se considerar a germinação $(\mathrm{G})$ e as plântulas normais em laboratório (PNL), os lotes I e II são semelhantes entre si e superiores ao lote III.

Os valores de condutividade elétrica (CE) dos lotes I e II, foram inferiores aos do lote III. Também, a CE diminuiu significativamente com o aumento do volume de água e aumentou com o período de embebição. Com o aumento do volume de água, ocorre maior diluição e conse-quente a diminuição dos valores de condutivi-dade. A concentração de solutos se torna maior com o passar do período de embebição, aumentando os valores de $\mathrm{CE}$. A variação da $\mathrm{CE}$ com o número de sementes por amostra não foi significativa, possivelmente, por se ter variado o volume de água para embebição (Tabela 2).

$\mathrm{Na}$ Tabela 3, observa-se que para o volume de $75 \mathrm{ml}$ de água não há diferença significativa entre os lotes, quando se utilizou amostras de 25, 50 e 75 sementes, no período de seis horas de embebição. Para 12 e 18 horas de embebição, somente os valores de CE obtidos para 75 sementes, discriminou o lote III dos demais. Quando se utilizou amostras de 50 sementes por 24 horas de embebição, somente houve diferença significativa do lote III para o lote II e neste mesmo período, o lote III diferiu dos demais, com amostras de 75 sementes. Com 36 horas de embebição, observou-se diferença do lote III para os demais com 50 e 75 sementes. Até 36 horas de embebição, em momento algum, houve diferença significativa entre os lotes I e II. À 72 horas de embebição, ocorreu diferença significativa entre os três lotes, com amostras de 50 sementes. Com 25 sementes, apenas o lote II mostrou-se superior aos demais e com 75 sementes o lote III foi inferior aos outros dois lotes.

Com o volume de $100 \mathrm{ml}$ de água e 25 sementes, foram observadas diferenças entre os lotes apenas com 72 horas de embebição, onde o lote II foi superior ao lote I. Para amostras de 50 sementes, esta diferença deu-se com 36 horas, identificando o lote III como de qualidade inferior aos lotes I e II. No entanto, quando se utilizou 75 sementes, com 18 horas de embebição já foi possível discriminar o lote I, como superior ao lote III. Após 72 horas de embebição, com amostras de 75 sementes, foi possível detectar diferenças entre os lotes ava- liados, sendo que o lote II foi superior ao lote I e esse superior ao lote III (Tabela 3).

Quando as sementes foram colocadas para embeber em $125 \mathrm{ml}$ de água, somente foi possível observar diferença significativa, entre os lotes, após 18 horas de embebição e com 75 sementes. Nesta situação, o lote I diferiu apenas do lote III. Com 25 sementes, diferenças entre os lotes foram observadas apenas com 72 horas de embebição, onde o lote II mostrouse superior ao III. Com 50 sementes, a partir de 24 horas de embebição, o lote III mostrou-se inferior aos lotes I e II. No entanto, com 75 sementes e para 18, 24 e 36 horas de embebição, o lote III mostrou-se inferior aos outros dois, porém o lote II apresentou condutividade elétrica superior ao lote I, embora sem diferença estatística entre eles. Nesses períodos de embebição, os lotes II e III, também foram estatisticamente iguais. Com 72 horas de embebição e 75 sementes, os lotes I e II foram superiores ao lote III (Tabela 3). Embora não tenha sido encontrado na literatura trabalhos desta natureza com 


\begin{tabular}{|c|c|c|c|c|c|c|c|c|c|c|}
\hline \multirow{4}{*}{$\begin{array}{l}\text { Período de } \\
\text { embebição } \\
\text { (horas) }\end{array}$} & \multirow{4}{*}{$\begin{array}{c}\mathrm{N}^{\circ} \mathrm{de} \\
\text { sementes }\end{array}$} & \multicolumn{9}{|c|}{ Condutividade elétrica ( $\mu \mathrm{mhos} / \mathrm{cm} / \mathrm{g}$ ) } \\
\hline & & \multicolumn{9}{|c|}{ Volume de água $(\mathrm{ml})$} \\
\hline & & \multicolumn{3}{|c|}{75} & \multicolumn{3}{|c|}{100} & \multicolumn{3}{|c|}{125} \\
\hline & & Lote I & Lote II & Lote III & Lote I & Lote II & Lote III & Lote I & Lote II & Lote III \\
\hline 6 & $\begin{array}{l}25 \\
50 \\
75\end{array}$ & $\begin{array}{l}74,9 \text { a } \\
62,3 \text { a } \\
52,1 \text { a }\end{array}$ & $\begin{array}{l}59,7 \mathrm{a} \\
54,8 \mathrm{a} \\
59,2 \mathrm{a}\end{array}$ & $\begin{array}{l}70,9 \mathrm{a} \\
62,3 \mathrm{a} \\
77,1 \mathrm{a}\end{array}$ & $\begin{array}{l}61,8 \mathrm{a} \\
47,8 \mathrm{a} \\
39,1 \mathrm{a}\end{array}$ & $\begin{array}{l}51,7 \mathrm{a} \\
47,3 \mathrm{a} \\
30,1 \mathrm{a}\end{array}$ & $\begin{array}{l}52,8 \mathrm{a} \\
50,8 \mathrm{a} \\
54,9 \mathrm{a}\end{array}$ & $\begin{array}{l}41,4 \mathrm{a} \\
36,4 \mathrm{a} \\
32,3 \mathrm{a}\end{array}$ & $\begin{array}{l}43,0 \mathrm{a} \\
35,5 \mathrm{a} \\
46,1 \mathrm{a}\end{array}$ & $\begin{array}{l}36,3 \mathrm{a} \\
43,1 \mathrm{a} \\
41,9 \mathrm{a}\end{array}$ \\
\hline 12 & $\begin{array}{l}25 \\
50 \\
75\end{array}$ & $\begin{array}{r}113,7 \mathrm{a} \\
90,5 \mathrm{a} \\
75,9 \mathrm{a}\end{array}$ & $\begin{array}{l}81,9 \mathrm{a} \\
81,4 \mathrm{a} \\
87,2 \mathrm{a}\end{array}$ & $\begin{array}{l}113,7 \mathrm{a} \\
102,4 \mathrm{a} \\
131,8 \mathrm{~b}\end{array}$ & $\begin{array}{l}91,9 \mathrm{a} \\
70,5 \mathrm{a} \\
59,4 \mathrm{ab}\end{array}$ & $\begin{array}{l}77,4 \mathrm{a} \\
73,4 \mathrm{a} \\
47,1 \mathrm{a}\end{array}$ & $\begin{array}{l}83,5 \mathrm{a} \\
81,9 \mathrm{a} \\
88,6 \mathrm{~b}\end{array}$ & $\begin{array}{l}63,8 \mathrm{a} \\
55,5 \mathrm{a} \\
49,5 \mathrm{a}\end{array}$ & $\begin{array}{l}63,9 \mathrm{a} \\
57,2 \mathrm{a} \\
71,7 \mathrm{a}\end{array}$ & $\begin{array}{l}66,0 \text { a } \\
74,1 \text { a } \\
70,8 \text { a }\end{array}$ \\
\hline 18 & $\begin{array}{l}25 \\
50 \\
75\end{array}$ & $\begin{array}{r}139,2 \mathrm{a} \\
114,7 \mathrm{a} \\
95,6 \mathrm{a}\end{array}$ & $\begin{array}{l}124,9 \mathrm{a} \\
105,9 \mathrm{a} \\
117,8 \mathrm{a}\end{array}$ & $\begin{array}{l}131,4 \mathrm{a} \\
147,7 \mathrm{a} \\
185,9 \mathrm{~b}\end{array}$ & $\begin{array}{r}109,8 \mathrm{a} \\
92,8 \mathrm{a} \\
86,2 \mathrm{a}\end{array}$ & $\begin{array}{l}96,9 \mathrm{a} \\
99,5 \mathrm{a} \\
66,0 \mathrm{a}\end{array}$ & $\begin{array}{l}101,4 \mathrm{a} \\
117,5 \mathrm{a} \\
134,7 \mathrm{~b}\end{array}$ & $\begin{array}{l}75,9 \mathrm{a} \\
74,0 \mathrm{a} \\
68,1 \mathrm{a}\end{array}$ & $\begin{array}{c}75,67 \mathrm{a} \\
75,4 \mathrm{a} \\
100,7 \mathrm{ab}\end{array}$ & $\begin{array}{r}83,0 \mathrm{a} \\
111,2 \mathrm{a} \\
111,7 \mathrm{~b}\end{array}$ \\
\hline 24 & $\begin{array}{l}25 \\
50 \\
75\end{array}$ & $\begin{array}{l}161,4 \mathrm{a} \\
136,7 \mathrm{ab} \\
113,7 \mathrm{a}\end{array}$ & $\begin{array}{l}139,8 \mathrm{a} \\
116,0 \mathrm{a} \\
130,3 \mathrm{a}\end{array}$ & $\begin{array}{l}154,9 \mathrm{a} \\
174,4 \mathrm{~b} \\
224,6 \mathrm{~b}\end{array}$ & $\begin{array}{r}117,3 \mathrm{a} \\
103,4 \mathrm{a} \\
92,42 \mathrm{a}\end{array}$ & $\begin{array}{r}105,4 \mathrm{a} \\
110,5 \mathrm{a} \\
71,2 \mathrm{a}\end{array}$ & $\begin{array}{l}108,1 \mathrm{a} \\
140,1 \mathrm{a} \\
155,3 \mathrm{~b}\end{array}$ & $\begin{array}{l}86,5 \mathrm{a} \\
83,5 \mathrm{a} \\
76,5 \mathrm{a}\end{array}$ & $\begin{array}{c}82,4 \mathrm{a} \\
83,6 \mathrm{a} \\
107,3 \mathrm{ab}\end{array}$ & $\begin{array}{r}95,8 \mathrm{a} \\
133,9 \mathrm{~b} \\
129,5 \mathrm{~b}\end{array}$ \\
\hline 36 & $\begin{array}{l}25 \\
50 \\
75\end{array}$ & $\begin{array}{l}179,5 \mathrm{a} \\
160,0 \mathrm{a} \\
134,4 \mathrm{a}\end{array}$ & $\begin{array}{l}157,9 \mathrm{a} \\
134,7 \mathrm{a} \\
151,4 \mathrm{a}\end{array}$ & $\begin{array}{l}182,8 \mathrm{a} \\
229,5 \mathrm{~b} \\
295,0 \mathrm{~b}\end{array}$ & $\begin{array}{l}146,4 \mathrm{a} \\
120,1 \mathrm{a} \\
112,8 \mathrm{a}\end{array}$ & $\begin{array}{r}118,6 \mathrm{a} \\
131,2 \mathrm{a} \\
85,3 \mathrm{a}\end{array}$ & $\begin{array}{l}129,7 \mathrm{a} \\
180,2 \mathrm{~b} \\
195,6 \mathrm{~b}\end{array}$ & $\begin{array}{r}98,6 \mathrm{a} \\
102,3 \mathrm{a} \\
93,3 \mathrm{a}\end{array}$ & $\begin{array}{r}93,1 \mathrm{a} \\
98,3 \mathrm{a} \\
128,5 \mathrm{ab}\end{array}$ & $\begin{array}{l}114,3 \mathrm{a} \\
177,3 \mathrm{~b} \\
167,5 \mathrm{~b}\end{array}$ \\
\hline 72 & $\begin{array}{l}25 \\
50 \\
75\end{array}$ & $\begin{array}{l}223,1 \mathrm{~b} \\
186,8 \mathrm{~b} \\
152,8 \mathrm{a}\end{array}$ & $\begin{array}{l}176,7 \mathrm{a} \\
141,3 \mathrm{a} \\
165,6 \mathrm{a}\end{array}$ & $\begin{array}{l}222,9 \mathrm{~b} \\
285,3 \mathrm{c} \\
377,8 \mathrm{~b}\end{array}$ & $\begin{array}{l}176,2 \mathrm{~b} \\
138,3 \mathrm{a} \\
148,2 \mathrm{~b}\end{array}$ & $\begin{array}{r}124,4 \mathrm{a} \\
138,5 \mathrm{a} \\
90,2 \mathrm{a}\end{array}$ & $\begin{array}{l}148,4 \mathrm{ab} \\
228,8 \mathrm{~b} \\
260,0 \mathrm{c}\end{array}$ & $\begin{array}{l}118,0 \mathrm{ab} \\
120,4 \mathrm{a} \\
119,5 \mathrm{a}\end{array}$ & $\begin{array}{l}101,3 \mathrm{a} \\
101,0 \mathrm{a} \\
141,9 \mathrm{a}\end{array}$ & $\begin{array}{l}149,2 \mathrm{~b} \\
230,8 \mathrm{~b} \\
221,2 \mathrm{~b}\end{array}$ \\
\hline
\end{tabular}

Médias seguidas pela mesma letra, para cada combinação período de embebição - número de sementes, em cada volume de água, não diferem significativamente entre si pelo teste Tukey, $0,1 \%$ (DMS $=40,639)$.

sementes florestais, verifica-se que é possível encontrar uma combinação ideal entre os fatores estudados e que permitem uma adequada diferenciação entre lotes de sementes. Trabalhando com sementes de tomate, Rodo et al. (1998) observaram que a melhor combinação número de sementes-volume de água, foi 25 sementes e $50 \mathrm{ml}$ de água.

Estes resultados podem ser reforçados pela análise das Figuras 1, 2 e 3. Pelas Figuras 1A, B C, referentes ao volume de $75 \mathrm{ml}$ de água deionizada, verifica-se que com 25 sementes (Figura 1A), não houve separação dos lotes I e III. Contudo, com 50 (Figura 1B) e 75 sementes (Figura 1C), o lote III foi identificado como de qualidade inferior aos dois outros. Essa discriminação entre os lotes, se dá de maneira mais nítida a partir de 24 horas de embebição.

Quando se fixa o volume de água em 100ml (Figuras 2A, B e C) os resultados encontrados são, de certa forma, contraditórios aos obtidos nas demais situações. Com 25 sementes, em geral, os três lotes apresentaram-se com comportamento semelhantes. Com 50 e 75 sementes, o lote III mostrou-se inferior aos demais, a partir de 36 horas, quando se utilizou 50 sementes e a partir de 18 horas quando se trabalhou com 75 sementes. Os lotes I e II, mostraram-se semelhantes, embora o lote II, tenha apresentado valores de condutividade elétrica superior ao lote I, quando se utilizou amostras de 50 sementes.

Variando-se o número de sementes a $125 \mathrm{ml}$ de água (Figuras 3A, B e C), verificou-se que com 25 e 50 sementes, os lotes I e II, mostraram-se semelhantes e superiores ao lote III. Para 75 sementes, o lote I apresentou menores valores de condutividade elétrica do que o lote II e esse, menores valores do que o lote III. Estes resultados são contraditórios aos obtidos pelos testes de germinação, em laboratório e em viveiro, onde o lote II apresentou melhor qualidade do que os demais.

$\mathrm{Na}$ Tabela 4, encontram-se as estimativas dos coeficientes 

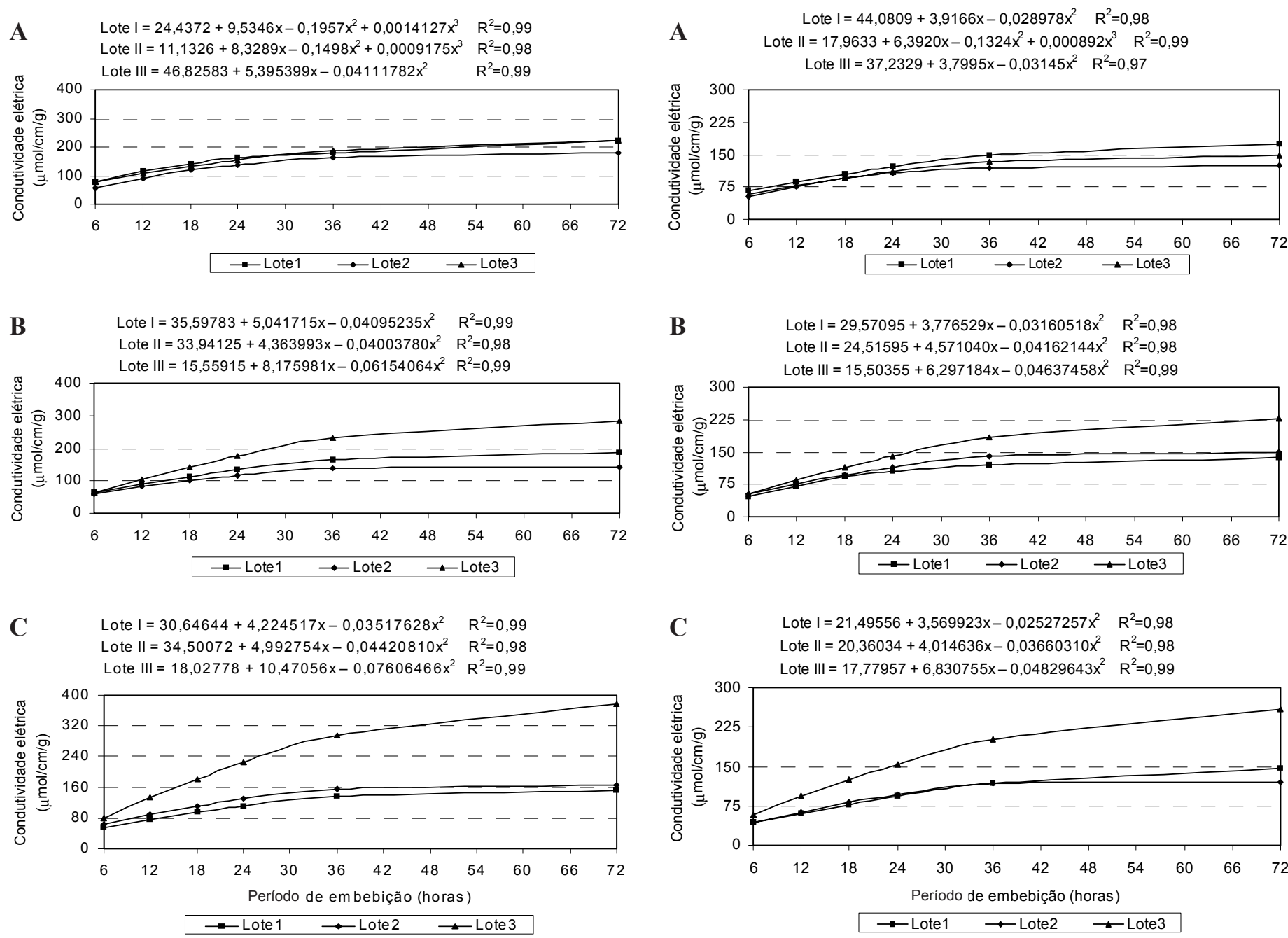

FIG. 1. Condutividade elétrica ( $\mu \mathrm{mhos} / \mathrm{cm} / \mathrm{g})$ de três lotes de sementes de jacarandá-da-bahia em amostras com 25 sementes (A), 50 sementes (B) e 75 sementes (C), embebidas em $75 \mathrm{ml}$ de água deionizada, por diferentes períodos de embebição.

Na Tabela 4, encontram-se as estimativas dos coeficientes de correlação simples entre os valores de condutividade elétrica e as características avaliadas nos testes de germinação, em laboratório e em viveiro. Observa-se que, quando se utilizou $75 \mathrm{ml}$ de água e 25 sementes, não houve diferença significativa nos coeficientes de correlação entre a condutividade elétrica e as características avaliadas nos testes de germinação, em laboratório e em viveiro, o mesmo acontecendo quando se utilizou 25 sementes e $100 \mathrm{ml}$ de água.

De uma forma geral, houve significância nos coeficientes de correlação entre a condutividade elétrica e os resultados de germinação, em laboratório e em viveiro, ou quando

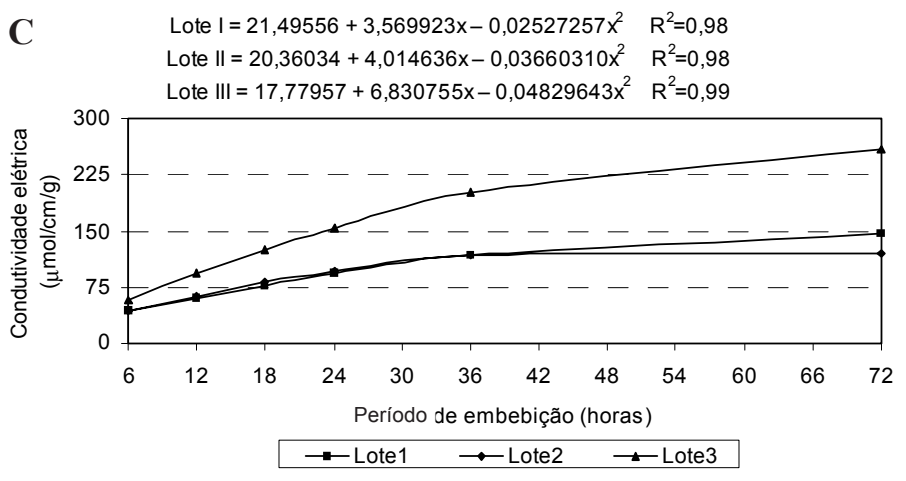

FIG. 2. Condutividade elétrica $(\mu \mathrm{mhos} / \mathrm{cm} / \mathrm{g})$ de três lotes de sementes de jacarandá-da-bahia em amostras com 25 sementes (A), 50 sementes (B) e 75 sementes (C), embebidas em $100 \mathrm{ml}$ de água deionizada, por diferentes períodos de embebição.

se aumentou o número de sementes, ou quando se aumentou o período de embebição, independente do volume de água utilizado para a embebição das sementes.

Considerando-se que, em muitos casos, o número de sementes é limitante para as sementes florestais e, de acordo com os resultados obtidos pode-se recomendar que o teste de condutividade elétrica, para sementes de jacarandá-da-bahia, seja conduzido com 50 sementes, em $75 \mathrm{ml}$ de água e com período de embebição igual ou superior a 36 horas, por permitir boa discriminação entre os lotes e alta associação com os resultados de germinação, tanto em laboratório quanto em viveiro. 

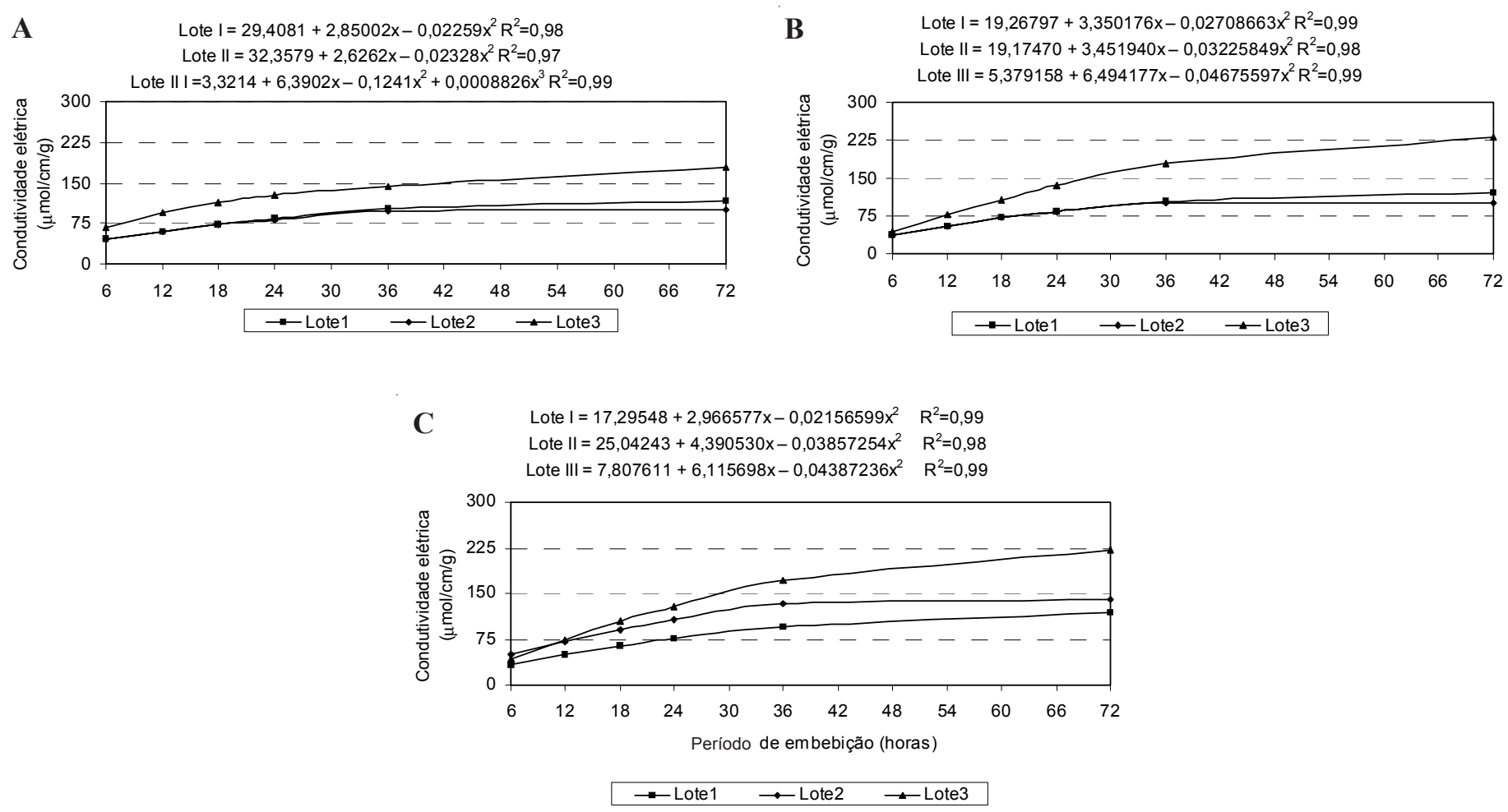

FIG. 3. Condutividade elétrica $(\mu \mathrm{mhos} / \mathrm{cm} / \mathrm{g})$ de três lotes de sementes de jacarandá-da-bahia em amostras com 25 sementes (A), 50 sementes (B) e 75 sementes (C), embebidas em 125ml de água deionizada, por diferentes épocas de embebição.

TABELA 4. Coeficientes de correlação simples entre os valores de condutividade elétrica e as características avaliadas em laboratório e em viveiro, para sementes de jacarandá-da-bahia, pertencentes a três lotes.

\begin{tabular}{|c|c|c|c|c|c|c|c|c|c|}
\hline \multirow{2}{*}{$\begin{array}{c}\text { Volume } \\
\text { de água }(\mathrm{ml})\end{array}$} & \multirow{2}{*}{$\begin{array}{c}\mathrm{N}^{\circ} \text { de } \\
\text { sementes }\end{array}$} & \multirow{2}{*}{$\begin{array}{c}\text { Período de } \\
\text { embebição (horas) }\end{array}$} & \multicolumn{7}{|c|}{ Coeficientes de correlação } \\
\hline & & & $\mathrm{PC}(\%)$ & $\mathrm{G}(\%)$ & PNL (\%) & IVG & $\mathrm{E}(\%)$ & PNV (\%) & IVE \\
\hline \multirow{18}{*}{75} & \multirow{6}{*}{25} & 6 & $0,24^{\mathrm{NS}}$ & $0,22^{\mathrm{NS}}$ & $0,15^{\mathrm{NS}}$ & $0,28^{\mathrm{NS}}$ & $0,21^{\mathrm{NS}}$ & $0,33^{\mathrm{NS}}$ & $0,38^{\mathrm{NS}}$ \\
\hline & & 12 & $0,38^{\mathrm{NS}}$ & $0,34^{\mathrm{NS}}$ & $0,33^{\mathrm{NS}}$ & $0,41^{\mathrm{NS}}$ & $0,38^{\mathrm{NS}}$ & $0,41^{\mathrm{NS}}$ & $0,43^{\mathrm{NS}}$ \\
\hline & & 18 & $0,02^{\mathrm{NS}}$ & $0,00^{\mathrm{NS}}$ & $0,05^{\mathrm{NS}}$ & $0,05^{\mathrm{NS}}$ & $0,04^{\mathrm{NS}}$ & $0,18^{\mathrm{NS}}$ & $0,21^{\mathrm{NS}}$ \\
\hline & & 24 & $0,12^{\mathrm{NS}}$ & $0,10^{\mathrm{NS}}$ & $0,05^{\mathrm{NS}}$ & $0,15^{\mathrm{NS}}$ & $0,14^{\mathrm{NS}}$ & $0,26^{\mathrm{NS}}$ & $0,30^{\mathrm{NS}}$ \\
\hline & & 36 & $0,26^{\mathrm{NS}}$ & $0,24^{\mathrm{NS}}$ & $0,19^{\mathrm{NS}}$ & $0,27^{\mathrm{NS}}$ & $0,27^{\mathrm{NS}}$ & $0,40^{\mathrm{NS}}$ & $0,43^{\mathrm{NS}}$ \\
\hline & & 72 & $0,39^{\mathrm{NS}}$ & $0,41^{\mathrm{NS}}$ & $0,30^{\mathrm{NS}}$ & $0,43^{\mathrm{NS}}$ & $0,34^{\mathrm{NS}}$ & $0,45^{\mathrm{NS}}$ & $0,49^{\mathrm{NS}}$ \\
\hline & \multirow{6}{*}{50} & 6 & $0,25^{\mathrm{NS}}$ & $0,30^{\mathrm{NS}}$ & $0,21^{\mathrm{NS}}$ & $0,29^{\mathrm{NS}}$ & $0,17^{\mathrm{NS}}$ & $0,23^{\mathrm{NS}}$ & $0,27^{\mathrm{NS}}$ \\
\hline & & 12 & $0,47^{\mathrm{NS}}$ & $0,51^{\mathrm{NS}}$ & $0,45^{\mathrm{NS}}$ & $0,50^{\mathrm{NS}}$ & $0,39^{\mathrm{NS}}$ & $0,44^{\mathrm{NS}}$ & $0,47^{\mathrm{NS}}$ \\
\hline & & 18 & $0,62 *$ & $0,62 *$ & $0,60 *$ & $0,63 *$ & $0,53 *$ & $0,61 *$ & $0,63 *$ \\
\hline & & 24 & $0,71 * *$ & $0,72 * *$ & $0,69 *$ & $0,73 * *$ & $0,64 *$ & $0,69 *$ & $0,71 * *$ \\
\hline & & 36 & $0,81 * *$ & $0,82 * *$ & $0,79 * *$ & $0,83 * *$ & $0,75^{* *}$ & $0,78 * *$ & $0,80 * *$ \\
\hline & & 72 & $0,88 * *$ & $0,90 * *$ & $0,86 * *$ & $0,90 * *$ & $0,82 * *$ & $0,83 * *$ & $0,84 * *$ \\
\hline & \multirow{6}{*}{75} & 6 & $0,52^{\mathrm{NS}}$ & $0,42^{\mathrm{NS}}$ & $0,49^{\mathrm{NS}}$ & $0,48^{\mathrm{NS}}$ & $0,52^{\mathrm{NS}}$ & $0,55^{\mathrm{NS}}$ & $0,50^{\mathrm{NS}}$ \\
\hline & & 12 & $0,69 *$ & $0,59 *$ & $0,67 * *$ & $0,65 *$ & $0,68 *$ & $0,70 *$ & $0,66 *$ \\
\hline & & 18 & $0,74 * *$ & $0,62 *$ & $0,69 *$ & $0,69 *$ & $0,71 * *$ & $0,75 * *$ & $0,71 * *$ \\
\hline & & 24 & $0,79 * *$ & $0,67 *$ & $0,75 * *$ & $0,74 * *$ & $0,76 * *$ & $0,78 * *$ & $0,74 * *$ \\
\hline & & 36 & $0,83 * *$ & $0,72 * *$ & $0,80 * *$ & $0,79 * *$ & $0,81 * *$ & $0,83 * *$ & $0,80 * *$ \\
\hline & & 72 & $0,86 * *$ & $0,76 * *$ & $0,83 * *$ & $0,82 * *$ & $0,84 * *$ & $0,85 * *$ & $0,82 * *$ \\
\hline
\end{tabular}




\begin{tabular}{|c|c|c|c|c|c|c|c|c|c|}
\hline \multicolumn{10}{|c|}{...Continuação Tabela 4} \\
\hline \multirow{2}{*}{$\begin{array}{c}\text { Volume } \\
\text { de água (ml) }\end{array}$} & \multirow{2}{*}{$\begin{array}{c}\mathrm{N}^{\circ} \mathrm{de} \\
\text { sementes }\end{array}$} & \multirow{2}{*}{$\begin{array}{c}\text { Período de } \\
\text { embebição (horas) }\end{array}$} & \multicolumn{7}{|c|}{ Coeficientes de correlação } \\
\hline & & & $\mathrm{PC}(\%)$ & $\mathrm{G}(\%)$ & PNL (\%) & IVG & $\mathrm{E}(\%)$ & PNV (\%) & IVE \\
\hline \multirow{18}{*}{100} & \multirow{6}{*}{25} & 6 & $0,18^{\mathrm{NS}}$ & $0,21^{\mathrm{NS}}$ & $0,24^{\mathrm{NS}}$ & $0,15^{\mathrm{NS}}$ & $0,03^{\mathrm{NS}}$ & $0,03^{\mathrm{NS}}$ & $0,00^{\mathrm{NS}}$ \\
\hline & & 12 & $0,03^{\mathrm{NS}}$ & $0,09^{\mathrm{NS}}$ & $0,08^{\mathrm{NS}}$ & $0,00^{\mathrm{NS}}$ & $0,14^{\mathrm{NS}}$ & $0,10^{\mathrm{NS}}$ & $0,12^{\mathrm{NS}}$ \\
\hline & & 18 & $0,05^{\mathrm{NS}}$ & $0,11^{\mathrm{NS}}$ & $0,12^{\mathrm{NS}}$ & $0,03^{\mathrm{NS}}$ & $0,11^{\mathrm{NS}}$ & $0,11^{\mathrm{NS}}$ & $0,13^{\mathrm{NS}}$ \\
\hline & & 24 & $0,08^{\mathrm{NS}}$ & $0,10^{\mathrm{NS}}$ & $0,14^{\mathrm{NS}}$ & $0,08^{\mathrm{NS}}$ & $0,07^{\mathrm{NS}}$ & $0,03^{\mathrm{NS}}$ & $0,05^{\mathrm{NS}}$ \\
\hline & & 36 & $0,01^{\mathrm{NS}}$ & $0,13^{\mathrm{NS}}$ & $0,09^{\mathrm{NS}}$ & $0,01^{\mathrm{NS}}$ & $0,13^{\mathrm{NS}}$ & $0,11^{\mathrm{NS}}$ & $0,15^{\mathrm{NS}}$ \\
\hline & & 72 & $0,03^{\mathrm{NS}}$ & $0,70^{\mathrm{NS}}$ & $0,06^{\mathrm{NS}}$ & $0,06^{\mathrm{NS}}$ & $0,14^{\mathrm{NS}}$ & $0,15^{\mathrm{NS}}$ & $0,18^{\mathrm{NS}}$ \\
\hline & \multirow{6}{*}{50} & 6 & $0,22^{\mathrm{NS}}$ & $0,18^{\mathrm{NS}}$ & $0,20^{\mathrm{NS}}$ & $0,23^{\mathrm{NS}}$ & $0,28^{\mathrm{NS}}$ & $0,32^{\mathrm{NS}}$ & $0,35^{\mathrm{NS}}$ \\
\hline & & 12 & $0,41^{\mathrm{NS}}$ & $0,36^{\mathrm{NS}}$ & $0,42^{\mathrm{NS}}$ & $0,41^{\mathrm{NS}}$ & $0,45^{\mathrm{NS}}$ & $0,45^{\mathrm{NS}}$ & $0,44^{\mathrm{NS}}$ \\
\hline & & 18 & $0,61 *$ & $0,55^{\mathrm{NS}}$ & $0,61 *$ & $0,59 *$ & $0,63 *$ & $0,63 *$ & $0,48 *$ \\
\hline & & 24 & $0,74 * *$ & $0,64 *$ & $0,72 * *$ & $0,71 * *$ & $0,76 * *$ & $0,76 * *$ & $0,77 * *$ \\
\hline & & 36 & $0,83 * *$ & $0,73 * *$ & $0,81 * *$ & $0,80 * *$ & $0,83 * *$ & $0,83 * *$ & $0,83 * *$ \\
\hline & & 72 & $0,93 * *$ & $0,85 * *$ & $0,91 * *$ & $0,91 * *$ & $0,93 * *$ & $0,93 * *$ & $0,92 * *$ \\
\hline & \multirow{6}{*}{75} & 6 & $0,62 *$ & $0,58 *$ & $0,65 *$ & $0,62 *$ & $0,61 *$ & $0,54^{\mathrm{NS}}$ & $0,55^{\mathrm{NS}}$ \\
\hline & & 12 & $0,69 *$ & $0,64 *$ & $0,71 * *$ & $0,68 *$ & $0,66 *$ & $0,62 *$ & $0,63 *$ \\
\hline & & 18 & $0,74 * *$ & $0,70 *$ & $0,76 * *$ & $0,72 * *$ & $0,72 * *$ & $0,67 *$ & $0,67 *$ \\
\hline & & 24 & $0,77 * *$ & $0,72 * *$ & $0,80 * *$ & $0,69 * *$ & $0,75 * *$ & $0,70 *$ & $0,70 *$ \\
\hline & & 36 & $0,79 * *$ & $0,75 * *$ & $0,83 * *$ & $0,75 * *$ & $0,78 * *$ & $0,73 * *$ & $0,73 * *$ \\
\hline & & 72 & $0,86 * *$ & $0,81 * *$ & $0,87 * *$ & $0,76 * *$ & $0,86 * *$ & $0,82 * *$ & $0,83 * *$ \\
\hline \multirow{18}{*}{125} & \multirow{6}{*}{25} & 6 & $0,64 *$ & 0,72 & $0,64 *$ & $0,65 *$ & $0,62 *$ & $0,53^{\mathrm{NS}}$ & $0,54^{\mathrm{NS}}$ \\
\hline & & 12 & $0,21^{\mathrm{NS}}$ & $0,07^{\mathrm{NS}}$ & $0,17^{\mathrm{NS}}$ & $0,18^{\mathrm{NS}}$ & $0,21^{\mathrm{NS}}$ & $0,25^{\mathrm{NS}}$ & $0,23^{\mathrm{NS}}$ \\
\hline & & 18 & $0,57^{\mathrm{NS}}$ & $0,42^{\mathrm{NS}}$ & $0,52^{\mathrm{NS}}$ & $0,53^{\mathrm{NS}}$ & $0,54^{\mathrm{NS}}$ & $0,57^{\mathrm{NS}}$ & $0,55^{\mathrm{NS}}$ \\
\hline & & 24 & $0,58 *$ & $0,53^{\mathrm{NS}}$ & $0,57^{\mathrm{NS}}$ & $0,58 *$ & $0,56^{\mathrm{NS}}$ & $0,51^{\mathrm{NS}}$ & $0,50^{\mathrm{NS}}$ \\
\hline & & 36 & $0,61 *$ & $0,55^{\mathrm{NS}}$ & $0,60 *$ & $0,61 *$ & $0,59 *$ & $0,53^{\mathrm{NS}}$ & $0,52^{\mathrm{NS}}$ \\
\hline & & 72 & $0,75 * *$ & $0,73 * *$ & $0,74 * *$ & $0,77 * *$ & $0,77 * *$ & $0,70 * *$ & $0,69 *$ \\
\hline & \multirow{6}{*}{50} & 6 & $0,36^{\mathrm{NS}}$ & $0,31^{\mathrm{NS}}$ & $0,28^{\mathrm{NS}}$ & $0,34^{\mathrm{NS}}$ & $0,35^{\mathrm{NS}}$ & $0,40^{\mathrm{NS}}$ & $0,41^{\mathrm{NS}}$ \\
\hline & & 12 & $0,45^{\mathrm{NS}}$ & $0,38^{\mathrm{NS}}$ & $0,37^{\mathrm{NS}}$ & $0,42^{\mathrm{NS}}$ & $0,43^{\mathrm{NS}}$ & $0,49^{\mathrm{NS}}$ & $0,50^{\mathrm{NS}}$ \\
\hline & & 18 & $0,62 * *$ & $0,54^{\mathrm{NS}}$ & $0,55^{\mathrm{NS}}$ & $0,59 * *$ & $0,58 * *$ & $0,65 * *$ & $0,65 * *$ \\
\hline & & 24 & $0,70 *$ & $0,63 *$ & $0,63 *$ & $0,67 *$ & $0,66 *$ & $0,72 * *$ & $0,71 * *$ \\
\hline & & 36 & $0,78 * *$ & $0,71 *$ & $0,72 * *$ & $0,75 * *$ & $0,74 * *$ & $0,79 * *$ & $0,78 * *$ \\
\hline & & 72 & $0,85 * *$ & $0,78 * *$ & $0,79 * *$ & $0,83 * *$ & $0,81 * *$ & $0,85 * *$ & $0,84 * *$ \\
\hline & \multirow{6}{*}{75} & 6 & $0,06^{\mathrm{NS}}$ & $0,04^{\mathrm{NS}}$ & $0,16^{\mathrm{NS}}$ & $0,02^{\mathrm{NS}}$ & $0,09^{\mathrm{NS}}$ & $0,08^{\mathrm{NS}}$ & $0,05^{\mathrm{NS}}$ \\
\hline & & 12 & $0,25^{\mathrm{NS}}$ & $0,15^{\mathrm{NS}}$ & $0,33^{\mathrm{NS}}$ & $0,17^{\mathrm{NS}}$ & $0,28^{\mathrm{NS}}$ & $0,27^{\mathrm{NS}}$ & $0,24^{\mathrm{NS}}$ \\
\hline & & 18 & $0,50^{\mathrm{NS}}$ & $0,40^{\mathrm{NS}}$ & $0,57^{\mathrm{NS}}$ & $0,42^{\mathrm{NS}}$ & $0,50^{\mathrm{NS}}$ & $0,51^{\mathrm{NS}}$ & $0,47^{\mathrm{NS}}$ \\
\hline & & 24 & $0,64 *$ & $0,55^{\mathrm{NS}}$ & $0,70 *$ & $0,58 *$ & $0,64 *$ & $0,64 *$ & $0,61 *$ \\
\hline & & 36 & $0,69 *$ & $0,62^{\mathrm{NS}}$ & $0,75 * *$ & $0,64 *$ & $0,69 *$ & $0,69 *$ & $0,66 *$ \\
\hline & & 72 & $0,85 * *$ & $0,80 * *$ & $0,88 * *$ & $0,82 * *$ & $0,85 * *$ & $0,86 * *$ & $0,85 * *$ \\
\hline
\end{tabular}




\section{CONCLUSÕES}

- O teste de condutividade elétrica se mostrou eficiente na diferenciação de lotes de sementes de Dalbergia nigra, apresentando alta associação com a germinação, em condições de laboratório e viveiro;

- o teste pode ser conduzido com amostras de 50 sementes, embebidas por pelo menos 36 horas em $75 \mathrm{ml}$ de água deionizada, a $25^{\circ} \mathrm{C}$.

\section{REFERÊNCIAS}

BARBEDO, C.J. \& CÍCERO, S.M. Utilização do teste de condutividade elétrica para previsão do potencial germinativo de sementes de ingá. Scientia Agricola, Piracicaba, v.55, n.2, p.249-259, 1998

BILIA, D.A.C.; MARCOS-FILHO, J.M. \& NOVEMBRE, A.D.L.C. Conservação da qualidade fisiológica de sementes de Inga uruguensis Hook. et Arn. Revista Brasileira de Sementes, Brasília, v.20, n.1, p.5-17, 1998.

BONNER, F.T. Testing tree seeds for vigor: a review. Seed Technology, Lawrence, v.20, n.1, p.5-17, 1998.

BONNER, F.T.; VOZZO, J.A.; ELAN, W.W. \& LAND-JR., S.B. Tree seed technology training course: student outline. New Orleans: U.S. Department of Agriculture, Forest Service,
Southern Forest Experiment Station, 1994. 81p. (General Technical Report, SO-107).

BRASIL. Ministério da Agricultura e da Reforma Agrária. Regras para análise de sementes. Brasília: SNDA/DNPV/CLAV, 1992. $365 \mathrm{p}$.

FERRAZ, I.D.K.; LIMA, V.N.S. \& COSTA, M.M. Testes de viabilidade em sementes de Carapa procera. In: SIMPÓSIO BRASILEIRO SOBRE TECNOLOGIA DE SEMENTES FLORESTAIS, 2. Atibaia, 1989. Anais. São Paulo: Instituto Florestal, 1991. p.39. (Série Documentos).

MAGUIRE, J.A. Speed of germination aid in selection and evaluation for seedling emergence and vigor. Crop Science, Madison, v.2, n.2, p.176-177, 1962.

OLIVEIRA, E.C. Morfologia de plântulas florestais. In: AGUIAR, I.B.; PIÑA-RODRIGUES, F.C.M. \& FIGLIOLIA, M.B. (coords.). Sementes florestais tropicais. Brasília: ABRATES, 1993. p.175-214.

PAULA, N.F.; BORGES, E.E.L.; BORGES, R.C.G. \& PAULA, R.C. Alterações fisiológicas em sementes de seringueira (Hevea brasiliensis Muell.Arg.) durante o armazenamento. Revista Brasileira de Sementes, Brasília, v.19, n.2, p.327-334, 1997.

RODO, A.B.; TILLMANN, M.A.A.; VILLELA, F.A. \& SAMPAIO, N.V. Teste de condutividade elétrica em sementes de tomate. Revista Brasileira de Sementes, Brasília, v.20, n.1, p.29-38, 1998. 